\title{
New Challenges for HDL-Modifying Therapies as a Strategy to Lower Cardiovascular Disease Events in Statin-Treated Patients
}

\author{
Editorial to: "Statin-Induced Decrease in ATP-Binding Cassette Transporter A1 Expression \\ via microRNA33 Induction may Counteract Cholesterol Efflux to High Density \\ Lipoprotein" by E.J. Niesor et al.
}

Robert S. Rosenson • H. Bryan Brewer Jr.

Published online: 24 February 2015

(C) Springer Science+Business Media New York 2015

High density lipoproteins (HDL) represent diverse subpopulations of particles that are heterogeneous in their physicochemical composition and functionality [1-3]. The cholesterol content of HDL particles, HDL cholesterol, has been used as a biomarker of atherosclerotic cardiovascular disease risk, and a target for pharmaceutical intervention [4]. Since many of these clinical trials were initiated, there have been transformational changes in our understanding of HDL biology and function such that increasing the cholesterol content of HDL is a failed therapeutic strategy $[3,4]$.

Among high-risk individuals treated with high-intensity statin therapy, low HDL-C has not been a consistent biomarker of cardiovascular events $[5,6]$. In the Treating to New Targets (TNT) trial, atorvastatin-treated patients who achieved low density lipoprotein (LDL) cholesterol levels less than $70 \mathrm{mg} / \mathrm{dL}$, recurrent cardiovascular events were higher among subgroups with low HDL cholesterol levels [7]. However, this association may have been confounded by the higher apolipoprotein $\mathrm{B}$, the major protein on atherogenic lipoproteins, in the subgroups of patients with low HDL cholesterol levels. Low HDL cholesterol is also considered a biomarker of elevated triglyceride-rich lipoproteins as shown in Mendelian randomization studies [9]. In contrast to HDL cholesterol, HDL

R. S. Rosenson $(\bowtie)$

Mount Sinai Heart, Mount Sinai Icahn School of Medicine, New

York, NY, USA

e-mail: robert.rosenson@mssm.edu

H. B. Brewer Jr.

MedStar Heart Institute, Washington Hospital Center,

Washington, DC, USA particle number is more strongly associated with increased cardiovascular risk in statin-treated individuals [6]. In JUPITER, a primary prevention trial of individuals at high risk for an initial cardiovascular event, on-trial levels of HDL cholesterol were not associated with cardiovascular risk in rosuvastatin-treated individuals [10]. In contrast, low ontrial levels of HDL particles were predictive of cardiovascular events.

Cholesteryl ester transfer protein (CETP) is a plasma protein that facilitates exchange of neutral lipid between lipoprotein particles resulting in lower concentrations of VLDL and LDL particles without necessarily changing HDL particle concentration with the exception of dalcetrapib that had modest effects of increasing HDL particle concentration [10]. In a Mendelian randomization study that included 16,503 cases and 46,576 controls, the CETP polymorphism rs3764261 on chromosome 16q13 were associated with higher levels of HDL cholesterol (3.86 mg/dL per allele) and lower levels of LDL cholesterol (-1.16 mg/dL per allele) [8]. Carriers of the rs3764261 trait had a $4 \%$ lower risk of myocardial infarction than non-carriers. Thus, CETP inhibition is a potential strategy for lowering cardiovascular events based on pharmacogenomics.

Two clinical outcomes trials have been completed with CETP inhibitors $[11,12]$. The ILLUSTRATE trial investigated the efficacy of the CETP inhibitor torcetrapib as a strategy to reduce the risk of recurrent events in atorvastatin-treated patients with stable coronary heart disease [11]. As compared with baseline values, torcetrapib increased HDL cholesterol by $72.1 \%$, and lowered LDL cholesterol and triglycerides by 24.9 and $9 \%$, respectively. Due to a higher mortality rate 
in torcetrapib-treated participants, this trial was terminated early. The adverse cardiovascular outcomes with torcetrapib were attributed to off-target toxicity that involved activation of the renin-angiotensin-aldosterone system. The dal-Outcomes trial compared the efficacy of the CETP modulator dalcetrapib in nearly 16,000 patients with recent acute coronary syndromes [12]. In the dalcetrapib group, $97 \%$ received statin therapy., Dalcetrapib-treated participants had an increase in HDL cholesterol during the trial that ranged from by 31 to $40 \%$, but neither baseline nor on-trial changes in HDL cholesterol were associated with the primary composite cardiovascular outcome. Although baseline LDL cholesterol levels were associated with future risk of cardiovascular events, dalcetrapib did not change LDL cholesterols from a baseline concentration of $76 \pm 26 \mathrm{mg} / \mathrm{dL}$. This trial was terminated early due to a lack of efficacy. On therapy with either dalcetrapib or placebo, there were no associations between baseline HDL cholesterol or apolipoprotein A-I and cardiovascular outcomes. In the dal-ACUTE trial, dalcetrapib improved macrophage cholesterol efflux but primarily via a non-ABCA1 pathway [13].

Despite the lack of clinical utility of HDL cholesterol and apolipoprotein A-I as bioimarkers of cardiovascular outcomes, CETP inhibition with dalcetrapib was shown to increase reverse cholesterol transport in an experimental animal model [14]. In hamsters injected with macrophageradiolabelled cholesterol, dalcetrapib administration increased plasma and fecal elimination of labeled sterol. However, these experiments were conducted in the absence of concomitant statin therapy.

In this issue, Niesor and colleagues investigate the effects of various statins on ABCA1 expression and macrophage cholesterol efflux to HDL [15]. Statins increase transcription of sterol element-binding protein (SREBP) and the expression of SREBP regulated genes that include microRNA33 (miR33). MicroRNAs are genomic encoded, short doublestranded RNAs that repress translation either through inhibition of ribosomal recognition and/or destabilization of mRNA by activation of RNA-induced silencing complex. MicroiRNA33 downregulates the expression of ABCA1 and ABCG1, and reduces HDL-mediated cholesterol efflux at the tissue level. In human THP-1 macrophages that were cholesterol loaded with acetylated LDL, incubation with atorvastatin $(1 \mu \mathrm{M})$ increased miR33 by $33 \%$ and reduced ABCA1 expression by $47 \%$. In mouse J7774A.1 macrophages loaded with ${ }^{3} \mathrm{H}$-cholesterol, ABCA1 mRNA and ABCA1-mediated cholesterol efflux were reduced with incubation by $1 \mu \mathrm{M}$ statin. The reduction in ABCA1 efflux was statin-specific ( simvastatin $>$ pitavastatin $>$ atorvastatin $>$ rosuvastatin $>$ pravastatin) and dose-dependent for simvastatin. Of additional interest is the potential tissue specificity of the inhibition of miR33 with minimum effect of statins on hepatic ABCA1 and potential major modulation on beta cell function and diabetes.
This study has important implications for our understanding of statin biology as well as the future development of HDL modifying therapies. Since ABCA1 is the essential pathway that mediates macrophage cholesterol efflux, and high potency statin therapy is evidence-based and guideline recommended therapy for high cardiovascular risk patients, Thus, the implications of this current report [15] may represent one source of residual cardiovascular risk in statin-treated patients. Interestingly, increased expression of hepatic miRNA33 [16] may be one explanation for the dose-dependent reduction in HDL cholesterol levels observed with atorvastatin [17] and differences in HDL cholesterol and HDL particle concentration reported with equipotent LDL cholesterol lowering doses of atorvastatin and rosuvastatin [6].

Future approaches for the development of new targets that reduce cardiovascular risk in statin-treated patients with HDL modifying therapies include inhibition of miR33, increase production of cholesterol deficient and phospholipid depleted apoA-I moieties that serve as ligands for ABCA1, and mobilization of cholesteryl ester from lipid-laden macrophages with LXR agonists [18].

\section{References}

1. Rosenson RS, Brewer Jr HB, Davidson WS, et al. Cholesterol efflux and atheroprotection: advancing the concept of reverse cholesterol transport. Circulation. 2012;125:1905-19.

2. Rosenson RS, Brewer Jr HB, Chapman J, et al. HDL measures, HDL particle heterogeneity and relation to atherosclerotic cardiovascular events. Clin Chem. 2011;57:392-410.

3. Rosenson RS, Brewer Jr HB, Ansell B, et al. Translation of highdensity lipoprotein function into clinical practice: current prospects and future challenges. Circulation. 2013;128:1256-67.

4. Medford R, Rosenson RS, Dagi TF, Offerman M. Biomarkers and sustainable innovation in cardiovascular drug development: lessons learned from near and far afield. Curr Atheroscler Rep. 2013;15:3219.

5. Rosenson RS, Underberg JA. Systematic review: evaluating the effect of lipid-lowering therapy on lipoprotein and lipid value. Cardiovasc Drugs Ther. 2013;27:465-79.

6. Rosenson RS, Davidson MH, Le N-A, Burke J, Pourfazib R. NgocAnh Le, Underappreciated opportunities for high-density lipoprotein particles in risk stratification and potential targets of therapy. Cardiovasc Drugs Ther. 2015;29:this issue.

7. Barter P, Gotto AM, LaRosa JC, et al. HDL cholesterol, very low levels of LDL cholesterol, and cardiovascular events. N Engl J Med. 2007;357:1301-10.

8. Voight BF, Peloso GM, Orho-Melander M, et al. Plasma HDL cholesterol and risk of myocardial infarction: a mendelian randomisation study. Lancet. 2012;380:572-80.

9. Rosenson RS, Davidson MH, Katheserin S, Hirsh B, Gaudet D. Triglyceride-rich lipoproteins and atherosclerosis. J Am Coll Cardiol. 2014;64:2525-40.

10. Mora S, Glynn RJ, Ridker PM. High-density lipoprotein cholesterol, size, particle number, and residual vascular risk after potent statin therapy. Circulation. 2013;128:1189-97. 
11. Barter PJ, Caulfield M, Eriksson M, et al. Effects of torcetrapib in patients at high risk for coronary events. N Engl J Med. 2007;357: 2109-22.

12. Schwartz GG, Olsson AG, Abt M, et al. Effects of dalcetrapib in patients with a recent acute coronary syndrome. N Engl J Med. 2012;367:2089-99.

13. Ray KK, Ditmarsch M, Kallend D, et al. The effect of cholesteryl ester transfer protein inhibition on lipids, lipoproteins, and markers of HDL function after an acute coronary syndrome: the dal-ACUTE randomized trial. Eur Heart J. 2014;35:1792-800.

14. Niesor EF, Magg C, Ogawa N, et al. Modulating cholesteryl ester transfer protein activity maintains efficient pre-beta-HDL formation and increases reverse cholesterol transport. J Lipid Res. 2010;51: $3443-54$.
15. Niesor EJ, Schwartz GG, Perez A, et al. Statin-induced decrease in ATP-binding cassette transporter A1 expression via microRNA33 induction may counteract cholesterol efflux to high-density lipoprotein. Cardiovasc Drugs Ther. 2015;29: this issue.

16. Cerda A, Hirata MH, Crespo Hirata RD. Molecular mechanisms underlying statin effects on genes involved in reverse cholesterol transport. Drug Metab Drug Interact. 2012;27:101-11.

17. Rosenson RS, Otvos JD, Hsia J. Effects of rosuvastatin and atorvastatin on low-density and high-density lipoprotein particle concentrations in patients with metabolic syndrome: a randomized, doubleblind, controlled study. Diabetes Care. 2009;32:1087-91.

18. van Capelleveen JC, Brewer HB, Kastelein JJ, Hovingh GK. Novel therapies focused on the high-density lipoprotein particle. Circ Res. 2014;114:193-204 